\title{
El abordaje de la salud sexual y reproductiva en mujeres inmigrantes desde un enfoque de género
}

\section{The approach to sexual and reproductive health in immigrant women from a gender perspective}

\section{A abordagem da saúde sexual e reprodutiva em mulheres imigrantes na perspectiva de gênero}

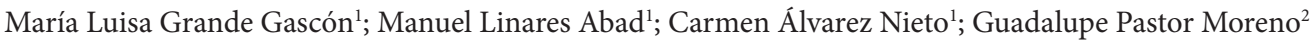

${ }^{1}$ Profesor/a de la Universidad de Jaén - ${ }^{2}$ Licenciada en Socióloga

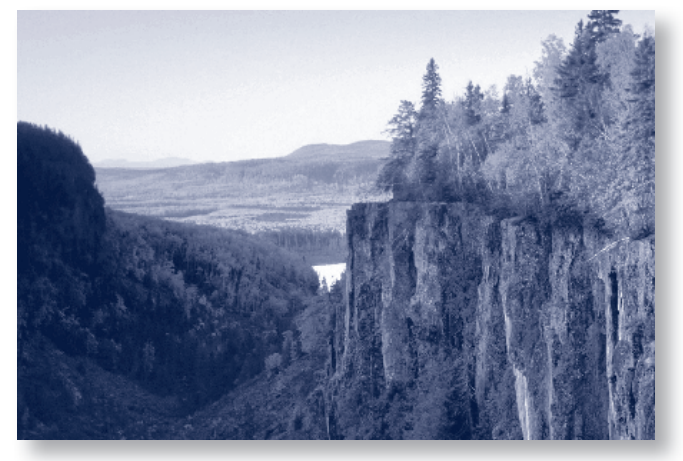

The approach to sexual and reproductive health in immigrant women from a gender perspective

\section{ABSTRACT}

In recent years there has occurred a significant increase in the immigrant population resident in Spain. And if at the beginning of phenomenon of migration they emigrated alone men, nowadays a growing number of women are migrating, or as result of family reunification, or as migration projects of themselves. This work aims to analyze, through a review of published literature, what are the factors that affect the sexual and reproductive health of immigrant women and what are the main barriers that they have on standardized access to the health services. In conclusion we can highlight that previous studies have shown that there are a number of factors that influence the sexual and reproductive health of female immigrants and that there is less use of health services for these, since there are multiple barriers that obstruct a normal integration in the public health system.

Key words: sexual and reproductive health, gender, immigrant women, cultural barriers.

A abordagem da saúde sexual e reprodutiva em mulheres imigrantes na perspectiva de gênero

\section{RESUMO}

Nos últimos anos tem-se observado importante implemento da população imigrante residente na Espanha. E se no início este era um fenômeno em que os homens emigravam, na atualidade está aumentando o número de mulheres que emigram, bem como a consequência da reagrupação familiar ou com projetos migratórios próprios. O objetivo do presente estudo é analisar, mediante uma revisão da bibliografia publicada, quais são os fatores que incidem na saúde sexual e reprodutiva das mulheres imigrantes e quais são as principais 
barreiras que têm no acesso aos serviços de saúde. Como conclusão, podemos destacar que nos estudos realizados destaca-se uma série de fatores que interferem na saúde sexual e reprodutiva das mulheres nesta condição e que têm menor utilização dos serviços de saúde, já que existem múltiplas barreiras que impedem uma integração normalizada no sistema de saúde público.

Palavras chave: Saúde sexual e reprodutiva; Gênero; Mulheres imigrantes; Barreiras culturais

\section{RESUMEN}

En los últimos años se ha producido un importante incremento de la población inmigrante residente en España. Y si en los inicios del fenómeno migratorio emigraban hombres solos, en la actualidad está aumentando el número de mujeres que emigran, bien como consecuencia de la reagrupación familiar o con proyectos migratorios propios. El objetivo de este trabajo es analizar, mediante una revisión de la bibliografía publicada, cuáles son los factores que inciden en la salud sexual y reproductiva de las mujeres inmigrantes y cuáles son las principales barreras que tienen en el acceso normalizado a los servicios sanitarios. Como conclusión podemos destacar que en los estudios realizados se ha puesto de manifiesto que existen una serie de factores que inciden en la salud sexual y reproductiva de las mujeres inmigrantes y que hay un menor uso de los servicios sanitarios por parte de éstas, ya que existen múltiples barreras que impiden una integración normalizada en el sistema sanitario público.

Palabras clave: salud sexual y reproductiva; género; mujeres inmigrantes; barreras culturales.

\section{INTRODUCCIÓN}

Los derechos relativos a la salud sexual y reproductiva tienen una influencia fundamental en las estrategias que se desarrollan para lograr la igualdad de género y el empoderamiento de las mujeres. Según el Fondo de Población de Naciones Unidas (FNUAP 2004), la salud sexual y reproductiva se define como "un estado de bienestar físico, mental y social y no de mera ausencia de enfermedad o dolencia, en todos los aspectos relacionados con el sistema reproductivo y sus funciones y procesos. Entraña además la capacidad de disfrutar de una vida sexual satisfactoria $y$ sin riesgos $y$ de procrear, y la libertad para decidir hacerlo o no hacerlo, cuándo y con qué frecuencia”.

En los últimos años han sido múltiples las conferencias internacionales que han abordado este tema y que han influido en los cambios que se han producido en este campo. La más referenciada es la Conferencia Internacional sobre Población y Desarrollo de Naciones Unidas celebrada en El Cairo en 1994, donde se marcaron las bases sobre la salud sexual y reproductiva y los derechos sexuales y reproductivos. Otro hito importante fue la IV Conferencia Mundial sobre la Mujer de Naciones Unidas celebrada en Beijing en 1995 en la que se reconoce el derecho de la mujer a controlar y decidir sobre las cuestiones relativas a su salud sexual y reproductiva.

El abordaje de la salud sexual y reproductiva ha evolucionado a lo largo del tiempo. Tradicionalmente la salud sexual y reproductiva estaba ligada de manera exclusiva al rol reproductivo, que tradicionalmente las mujeres han venido desempeñando en la sociedad. Así el término utilizado era salud materno-infantil. En la actualidad la salud sexual y reproductiva tiene una relación directa con la desigualdad y la discriminación que sufren las mujeres en la 
sociedad. El concepto de salud materno-infantil se sustituye por el de salud sexual y reproductiva (Espinosa 2005).

El Observatorio de salud de la mujer en un estudio denominado "Aproximación a la salud sexual y reproductiva en España" pone de manifiesto la necesidad de contemplar en las investigaciones sobre este tema los aspectos sociales, culturales y psicológicos para incluir la perspectiva de género, ya que van a influir en las relaciones afectivo-sexuales entre hombres y mujeres y sobre cómo los roles y las atribuciones social y culturalmente definidas determinan el grado de desigualdad de género existente. En este sentido en las conclusiones de este estudio se recoge que es importante el uso de una metodología cualitativa y cuantitativa que permitan conocer las pautas reproductivas y las prácticas sexuales que caracterizan una determinada sociedad y cultura.

En los últimos años se ha producido un importante incremento de la población inmigrante residente en España. Y si en los inicios del fenómeno migratorio emigraban hombres solos, en la actualidad está aumentando el número de mujeres que emigran, bien como consecuencia de la reagrupación familiar o con proyectos migratorios propios. Según el Padrón de Habitantes, a 1 de enero de 2009 la población extranjera residente en España es de 5.598.691, lo que supone un porcentaje de $12 \%$ sobre la población total de España. Por sexo, 2.973.707 son hombres y 2.624.984 son mujeres.

Con este trabajo se pretende analizar, mediante una revisión de la bibliografía publicada, cuáles son los factores que inciden en la salud sexual y reproductiva de las mujeres inmigrantes y cuáles son las principales barreras que tienen en el acceso normalizado a los servicios sanitarios.

\section{MATERIAL Y MÉTODOS}

Para realizar esta revisión se han analizado los artículos publicados desde el año 2000 en las siguientes bases de datos o plataformas de bases de datos: Scopus, Cuiden, MedLine, Isi Web of Knowledge y Cinahl. Las términos utilizados para la búsqueda han sido: "salud sexual y reproductiva" y "mujeres inmigrantes" para Cuiden y "sexual and reproductive health", “immigration" para las demás. Además se han analizado otros documentos y publicaciones que hacen referencia a la salud sexual y reproductiva de las mujeres inmigrantes, fundamentalmente aquellos donde se describe cómo debe ser la atención sanitaria recibida por éstas.

\section{DESARROLLO DEL TEMA}

Las mujeres inmigrantes residentes en España, mayoritariamente están en edad fértil y tienen unas necesidades específicas relacionadas en gran medida con la salud sexual y reproductiva (Oliver 2007). También presentan una serie de factores de riesgo sexual y reproductivo que en algunos estudios se consideran como predictivos (McDonald 2009)

Existe una menor visibilidad de los problemas de salud que presentan las mujeres inmigrantes. En este sentido hay que destacar que se han realizado pocos estudios sobre este tema. En todo caso la mayoría pone de manifiesto que existen una serie de factores que inciden en la salud sexual y reproductiva que está ligada a las desigualdades de género y a las condiciones socio-económicas que padecen las mujeres inmigrantes (Oliver 2007).

Por otra parte, las mujeres inmigrantes proceden de culturas diferentes y constituyen un colectivo muy heterogéneo, aunque desde los países receptores tendemos a verlas de manera homogénea. Existen diferencias cul- 


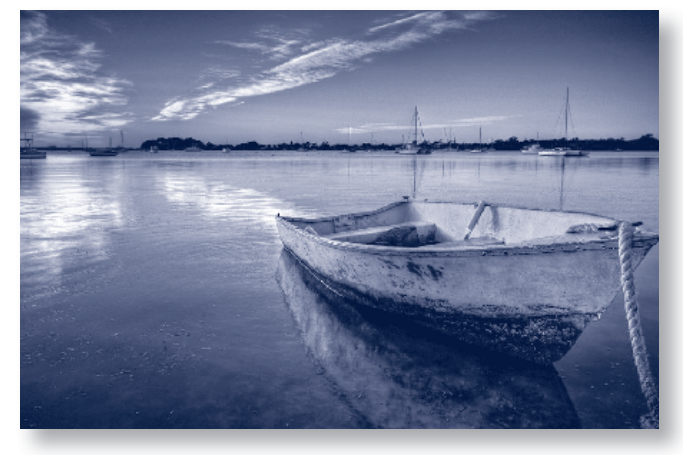

turales importantes que dependen del país de origen, de la zona geográfica de procedencia, de la religión que practiquen y de la etnia a la que pertenezcan. La cultura tiene una gran influencia sobre la concepción de la salud y la enfermedad de la población inmigrante, ya que ambas son construcciones sociales $y$, como tales, tienen diferentes significados e interpretaciones para cada grupo cultural. Cada cultura se enfrenta a las enfermedades de diferente manera, y construye su propio sistema de salud, más o menos elaborado, para abordarlas. Por lo tanto, en el caso de las mujeres inmigrantes, debemos considerar las creencias que tienen sobre la salud, la enfermedad, la curación, la muerte y las implicaciones familiares y sociales de la enfermedad (Roca 2001).

Las diferencias culturales se ponen de manifiesto en la concepción e interpretación que cada grupo étnico hace sobre la salud, la enfermedad y la muerte, lo que implica universos simbólicos distintos que condicionan las prácticas, los usos y las relaciones que se establecen entre los sujetos que participan en cada contexto (Canals 2004, Castillo et al 2001).

En este sentido, la marginación en la que viven y el escaso respeto a las diferencias culturales, dificulta la interacción y la comunicación entre las mujeres inmigrantes y los profesionales sanitarios. Esta situación es consecuencia de las distintas cosmovisiones de los pacientes y los profesionales que se mueven en marcos de referencia diferentes culturalmente (Rey et al 2004).

La mujer inmigrante, en un primer momento, se encuentra en lo que define Moreno (2006: 7) "una especie de limbo en el que ya no es lo que era, pero aún no es lo que quiere ser. Su forma de vida ha sido trastocada y sus representaciones simbólicas alteradas". Por lo tanto tiene que adaptarse a un entorno diferente que le es hostil, para ello tiene que elaborar de nuevo todas sus prácticas incorporando nuevas visiones y conductas e intentando retener de su propia cultura todo aquello que le es útil. La multiculturalidad hace necesario la aceptación de la diversidad de categorías culturales relacionadas con la salud y la enfermedad para integrar a las mujeres inmigrantes en el sistema sanitario (Roca 2001).

Por otra parte, según se establece la Ley orgánica $8 / 2000$, de 22 de diciembre, sobre derechos y libertades de los extranjeros en España y su integración social, en su artículo 12 (Derecho a la asistencia sanitaria), tienen derecho a la atención sanitaria la población extranjera que se encuentran inscrita en el padrón municipal en las mismas condiciones que los españoles; todas situaciones de urgencia por enfermedad grave o accidente; los menores de 18 años, cobertura de asistencial total; y las embarazadas durante el embarazo, parto y puerperio. Sin embargo, a pesar de que en teoría esto es así, en la práctica existen una serie de barreras que dificultan el acceso de los mismos a los servicios de salud.

Las mujeres inmigrantes que no están regularizadas y empadronadas quedan fuera de las prestaciones del sistema de salud, y sólo pueden hacer uso de los servicios de urgencias, no pudiendo acceder a una asistencia sanitaria normalizada. En gran medida, acceden al sistema de salud a través de los servicios de 
urgencias cuando presentan patologías y enfermedades que requieren de dicha atención. Utilizan en menor medida otro tipo de recursos, que puedan mejorar su nivel general de salud y su salud sexual y reproductiva (Sanz et al 2004).

En la actualidad desde el sistema público de salud se ofrecen programas relacionados con la salud sexual y reproductiva a los cuáles las mujeres inmigrantes acceden en menor medida que la población autóctona (Casamitjana 2009, Pardo 2006, de la Torre et al 2006). Así en un estudio realizado en Finlandia (Malin, 2009) se señala que entre las mujeres inmigrantes, hubo diferencias significativas en el tipo de atención materna recibida, presentando en algunos casos más problemas de salud durante el embarazo y el parto. En este sentido, otro estudio pone de manifiesto que existe un mayor porcentaje de mujeres inmigrantes que de mujeres autóctonas que presentan uno o más factores de riesgo durante el embarazo y el parto (Martínez et al 2004, Martínez et al 2002).

El menor uso de los servicios de salud también guarda relación con la existencia de múltiples barreras que impiden una integración normalizada en el sistema sanitario público. Markez (2006: 154), alude a la existencia de una limitación en el acceso a los servicios de salud por parte de la población inmigrante haciendo referencia a "aquellos elementos, internos o externos, que dificultan la atención a la salud de las personas que lo precisan". Distingue tres tipos de problemas: problemas asociados al sistema, problemas de comunicación y problemas asociados al paciente-inmigrante. Por su parte Lobato y Oliver (2004) establecen la existencia de cuatro tipo de barreras: barreras administrativas y normativas, barreras lingüísticas y de comunicación, barreras cultura- les y religiosas, y el rechazo pasivo del sistema o de los profesionales sanitarios.

Las diferencias culturales y lingüísticas suponen otra importante barrera en el uso de los servicios de salud (Valtueña 2000, Newbold 2009). Si bien el conflicto del idioma es algo fácil de solucionar a través de intérpretes, no es tan sencillo el problema de las diferencias culturales. La comunicación y las relaciones humanas se encuentran determinadas por la cultura, de modo tal que el vínculo que pueda establecerse entre los profesionales sanitarios locales y los pacientes inmigrantes no podrá mejorarse si no se tienen en cuenta las diferencias culturales que en muchos casos pueden dificultar el entendimiento entre ambos. Esto tiene como consecuencia dificultades en el diagnóstico, el tratamiento y la adherencia al tratamiento.

A todo esto hay que añadir que los profesionales de la salud forman parte de una sociedad en la que un porcentaje de la población rechaza o no acepta a la población inmigrante, presentando actitudes racistas y xenófobas. Así, la mayoría de las personas, y por ende una gran cantidad de profesionales sanitarios, no se declara abiertamente racista, pero tiene una serie de prejuicios y estereotipos que son asumidos sin ningún tipo de cuestionamiento.

Las consecuencias de lo anteriormente expuesto son graves, si las mujeres inmigrantes perciben un trato discriminatorio o no adecuado, dejan de acudir a los servicios de salud. Las formas que puede tomar esta discriminación son diversas (Moreno 2006): o bien se tiene una actitud de recelo hacia ellas, o bien se les trata con un excesivo paternalismo, considerando que hay que atenderlas por una cuestión de caridad. La mirada sospechosa y la mirada compasiva, tal y como señala Moreno, tienen como consecuencia la discriminación 
y la exclusión. Esto supone que en numerosas ocasiones, la inmigración es percibida por los profesionales sanitarios como un problema, y no como un fenómeno social más frente al que hay que hacer adaptaciones mutuas.

El desconocimiento de nuestro sistema de salud supone otra dificultad añadida para las mujeres inmigrantes, en la mayor parte de los casos provenientes de países donde el sistema de salud no es público, o si lo es, tiene prestaciones y coberturas muy pobres. Así, la inmigrante que lleva poco tiempo en nuestro país no conoce cuales son sus derechos en salud ni las prestaciones que le ofrece el sistema público de salud (Garcés-Palacio 2008, Kormosky 2008).

Todas estas situaciones producen inequidad en el acceso y uso de los servicios de salud. La mujer inmigrante, aún si está regularizada y tiene derecho a una cobertura sanitaria, se encuentra con una serie de limitaciones que no existen para la población autóctona y para las que todavía no se han encontrado soluciones globales y estructurales.

\section{BIBLIOGRAFÍA}

- Canals, J. (2004) La importancia de las dimensiones culturales en la asistencia y en la promoción de la salud. Servicios Sociales y Política Social 65, 9-20.

- Casamitjana, M.; Sala, M.; Ochoa, D.; Fusté, P.; Castells, X.; Alameda, F. (2009) Results of a cervical cancer screening programme from an area of Barcelona (Spain) with a large immigrant population. European Journal of $\mathrm{Pu}$ blic Health 19(5), 499-503.

- Castillo, S.; Mazarrasa, L.; Sanz, B. (2001) Mujeres inmigrantes hablando de su salud. Utilización de sus discursos como estrategia para promocionar su salud. Index de Enfermería 34, 9-14.

- De la Torre, J.; Coll, C. Coloma, M.; Martíon J.L.; Padrón, E.; González, N.L. (2006). Control de gestación en inmigrantes. Anales del Sistema sanitario de Navarra 29 supl.1, 49-61.
- Espinosa, J. (2005) Guía para programas y proyectos de salud sexual y reproductiva en África. Programa VITA. AECI, Madrid.

- FNUAP. (2004) Estado de la población mundial 2004. El consenso de El Cairo, diez años después: población, salud reproductiva y acciones mundiales para eliminar la pobreza. Disponible en: www.fnuap.org

- Garcés-Palacio, I.C.; Altarac, M.; Scarinci, I.C. (2008) Contraceptive knowlege and use among low-income Hispanic immigrant women and non-Hispanic women. Contraception. 77(4), 270-5.

- Kormosky, J.L.; Peck, J.D.; Sweeney, A.M.; Adelson, P.L.; Schantz, S.L. (2008) Reproductive characteristics of Southeast Asian immigrants before and after migration. Journal of Immigrant and Minority Health. 10(2), 13543.

- Lobato, P.; Oliver, B. (2004) Aspecto normativos y organizativos sobre atención sanitaria a inmigrantes en Andalucía. En: Lobato P. et al., eds., Manual de atención sanitaria a inmigrantes. Fundación Progreso y Salud. Consejería de Salud, Sevilla.

- Oliver, M.I.; Baraza, P.; Martínez, E. (2007) La salud y la mujer inmigrante. En: García R. Manual de atención sanitaria a inmigrantes. Consejería de Salud, Sevilla.

- OSM (Observatorio de Salud de la Mujer). Aproximación a la salud sexual y reproductiva en España. Disponible en:

http://www.msc.es/organizacion/sns/planCalidadSNS/ pdf/equidad/Estudio_salud_sexual_y_reproductiva._ Observatorio.pdf

- Malin, M.; Gissler, M. (2009) Maternal care and birth outcomes among ethnic minority women in Finland. BMC Public Health. Mar 20(9), 84.

- Markez, I. (2006) La salud mental de los inmigrantes: ni enfermos, ni delincuentes. Mediación e integración. En Markez, I., ed., Políticas de inmigración, interculturalidad y mediación. Respuestas a la exclusión. Gakoa Liburuak, San Sebastián.

- Martínez, E.; López, A.F. (2004) Diferencias de morbilidad asociada al embarazo y de resultados obstétricos y perinatales entre mujeres inmigrantes y autóctonas. Matronas Profesión 5(17), 12-18. 
- Martínez, M.F.; Monzón, A.; Falcón, M.A.; Pardo, A. (2002) Partos de inmigrantes y jóvenes inmigrantes en el Hospital Clínico Universitario de Zaragoza (España). Index de Enfermería 38, 15-17.

- McDonald, J.A.; Manlove, J.; Ikramullah, E.N. (2009) Inmigration measures and reproductive health among Hispanic youth: findings from the national longitudinal survey of youth, 1997-2003. Journal of Adolescent Health. 44(1), 14-24.

- Moreno, M. (2006). Del cuidado de la diversidad a la diversidad del cuidado. Index de Enfermería 55; 7-8.

- Newbold, K.B.; Willinsky, J. (2009) Providing family planning and reproductive healthcare to Canadian immigrants: perception of healthcare providers. Culture, Health and Sexuality 11(4), 369-82.
- Pardo, G.; Engel, J.L.; Agudo, S. (2006) Acercamiento a las características sociosanitarias de las mujeres subsaharianas de dos programas de atención a inmigrantes de Madrid. Enfermería Clínica. 16(5), 264-7.

- Rey, M.D.; Gálvez, A. (2004) Mitos vs vivencias de las mujeres inmigrantes; el embarazo, el parto y el puerperio. Una enfermería transcultural. Evidentia 1(3).

- Roca, N. (2001) Inmigración y salud. Situación actual en España. Revista ROL de Enfermería 24(10), 682-686.

- Sanz, B.; Castillo, S.; Mazarrasa, L. (2004) Uso de los servicios sanitarios en las mujeres inmigrantes de la Comunidad de Madrid. Metas Enfermería 7(1), 26-32.

- Valtueña, O. (2000) Medidas para proteger y promover la salud de los inmigrantes en España. Migraciones 8, 231-249.

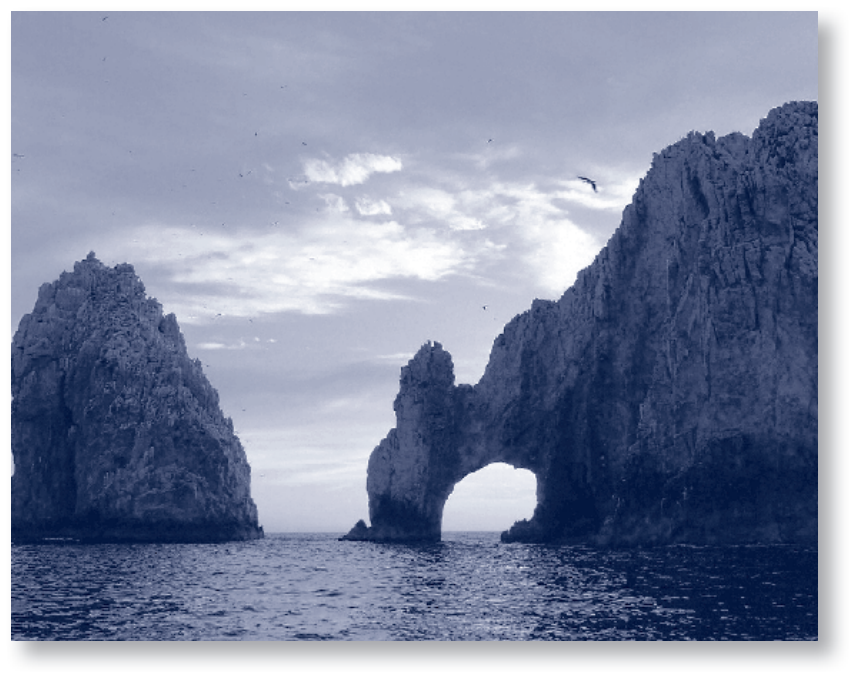

